\title{
Latent tuberculosis infection among patients with and without type-2 diabetes mellitus: results from a hospital case-control study in Atlanta
}

Argita D. Salindri ${ }^{*}$ (D) J. Sonya Haw², Genet A. Amere ${ }^{1}$, Joyce T. Alese ${ }^{1}$, Guillermo E. Umpierrez ${ }^{2}$ and Matthew J. Magee ${ }^{1,3}$

\begin{abstract}
Objective: The purpose of this study is to compare the prevalence of latent TB infection (LTBI) among patients with type-2 diabetes mellitus (T2DM) to healthy controls without T2DM. To achieve this objective, we conducted a casecontrol study in a large hospital in Atlanta from 2016 to 2019.

Results: We enrolled 98 cases; 119 potential controls were screened, 84 of which had $\mathrm{HbA} 1 \mathrm{C} \geq 5.7 \%$ and one did not have QFT result, leaving 34 (28.6\%) individuals enrolled as controls. LTBI prevalence was $9.2 \%$ among cases and $14.7 \%$ among controls (crude odds ratio $0.59,95 \% \mathrm{Cl} 0.19-2.04$ ). After adjusting for age and sex, the adjusted odds of LTBI among patients with T2DM was $0.45(95 \% \mathrm{Cl} 0.13,1.71)$ times the controls. We did not observe a statistically significant association between LTBI and T2DM. However, we reported a positive correlation between $\mathrm{HbA}$ 1c level and nil count among individuals with $L T B I\left(R^{2}=0.55, p<0.01\right)$. In addition, we reported a high prevalence of $L T B I$ among adults with T2DM and family members without T2DM.
\end{abstract}

Keywords: Tuberculosis, Type-2 diabetes, Latent TB infection

\section{Introduction}

Emerging cross-sectional evidence suggests type 2 diabetes mellitus (T2DM) is associated with higher prevalence of latent tuberculosis infection (LTBI) [1, 2]. Results from US national survey data reported $12 \%$ LTBI prevalence among adults with diabetes compared to 5\% LTBI prevalence among adults without diabetes [3, 4]. However, less is known regarding US regional differences in the relationship between LTBI and T2DM or the extent that the association is different in racial/ethnic subgroups [5].

\footnotetext{
*Correspondence: asalindri1@student.gsu.edu

${ }^{1}$ Division of Epidemiology and Biostatistics, Department of Population Health Sciences, School of Public Health, Georgia State University, Atlanta, GA 30303, USA

Full list of author information is available at the end of the article
}

Although T2DM is an established risk factor for tuberculosis (TB) disease, whether T2DM increases the risk of LTBI, or vice versa, remains a critical gap in knowledge $[2,6]$. Results from murine models of diabetes and TB suggest that phagocytosis and uptake of Mycobacterium tuberculosis $(M t b)$ by monocytes and macrophages are reduced in the context of diabetes [7]. Pro-inflammatory cytokine expression profiles associated with $M t b$ control [i.e., interferon (IFN)- $\gamma$ ] were also delayed in diabetic animal models [8]. These may support the premise that diabetes increases the risk of TB infection or reactivation from latency. Alternatively, LTBI may influence T2DM risk as increasing evidence indicates TB modulates human adipose tissue function and may impact host metabolic homeostasis [9]. For example, an animal model of nondiabetic Guinea pigs reported that an infection of

(c) The Author(s) 2021. This article is licensed under a Creative Commons Attribution 4.0 International License, which permits use, sharing, adaptation, distribution and reproduction in any medium or format, as long as you give appropriate credit to the original author(s) and the source, provide a link to the Creative Commons licence, and indicate if changes were made. The images or other third party material in this article are included in the article's Creative Commons licence, unless indicated otherwise in a credit line to the material. If material is not included in the article's Creative Commons licence and your intended use is not permitted by statutory regulation or exceeds the permitted use, you will need to obtain permission directly from the copyright holder. To view a copy of this licence, visit http://creativeco mmons.org/licenses/by/4.0/. The Creative Commons Public Domain Dedication waiver (http://creativecommons.org/publicdomain/ zero/1.0/) applies to the data made available in this article, unless otherwise stated in a credit line to the data. 
$M t b$ alone could result in glucose intolerance and incresead level of serum free fatty acid, two strong predictors of T2DM development [10].

To definitively establish whether LTBI increases diabetes risk or impact host metabolic outcomes will require large longitudinal studies and extensive followup time. Thus to gain preliminary insight into the LTBIT2DM relationship, we conducted a case-control study to (a) compare the prevalence of LTBI among patients with T2DM to controls without T2DM, and (b) assess whether glycated hemoglobin (HbA1c) level is correlated with QuantiFERON Gold-in-tube test (QFT) quantitative measures (i.e., antigen, mitogen, or nil count).

\section{Main text}

\section{Methods}

We conducted a case-control study in a large hospital in metro Atlanta, Georgia, US, from 2016 to 2019. Eligible cases were human immunodeficiency virus (HIV)-negative adults ( $\geq 21$ years) with newly diagnosed T2DM (diagnosed within the past 3 years) and no history of TB disease. Among cases, HbA1c (\%) was obtained from the electronic medical chart by abstracting the plasma HbA1c value closest to the date of study enrollment. Eligible controls included adult family members/friends of cases with $\mathrm{HbA} 1 \mathrm{c}<5.7 \%$ (measured at the time of screening by a point-of-care HbA1c [11], Siemens DCA Vantage Analyzer) and no self-reported prior diagnosis of pre-diabetes, T2DM, HIV, or TB disease. HbA1c values were categorized according to the American Diabetes Association classification with HbA1c $<5.7 \%$ considered as "normoglycemic", HbA1c 5.7-6.4\% as "pre-diabetes" and, HbA1c $\geq 6.5 \%$ as "diabetes" [12]. Enrolled participants had LTBI status determined by QFT Gold-InTube test at the time of study enrollement. QFT samples were prepared and processed following the manufacturer's (QIAGEN) guidelines. Results of the quantitative QFT measures were interepreted using three ciriteria: (a) mitogen-nil and tuberculin-nil values, (b) percentage of avian difference, and (c) percentage of tuberculin response [13], and classified as "positive", "negative", or "indeterminate" for TB infection. Cases and controls were excluded if they were using steroids or tumor necorosis factor (TNF)- $\alpha$ antagonist therapy at the time of screening, resided outside DeKalb or Fulton counties, or did not speak English. Participants clinical and demographic characteristics were obtained using study questionnaires and medical chart review.

\section{Data analysis}

We compared demographic and clinical characteristics of cases to controls using Chi-square and Fisher's Exact tests. Logistic regression was used to estimate the association between LTBI and T2DM quantified by odds ratios and $95 \%$ confidence intervals (CIs). Covariates included in the final adjusted model were selected purposively based on previously published literature and directed acyclic graph theory [14]. Among those with LTBI, linear regression was used to estimate the correlation between $\mathrm{HbA1c}$ and quantitative QFT measures. Linear regression models were used to measure the relationship between HbA1c and (a) nil count, (b) TB antigen-nil, and (c) mitogen-nil values. In addition to beta estimates and corresponding 95\% CI, we also reported $\mathrm{R}^{2}$ values or the coefficient of determination as a measure of how well the linear regression model described the observed data.

\section{Results}

We screened 199 potential eligible cases and 405 potential eligible controls during the study period. Among individuals screened, a total of 98 cases and 34 controls were enrolled. Of 199 eligible cases, 91 (45.7\%) refused to participate or were excluded (Additional file 1: Figure S1). Out of 108 cases enrolled, 10 were later excluded, leaving 98 (90.7\%) included in the final analyses. Among 405 potential eligible controls, 35 (12.2\%) self-reported prior diagnosis of pre-diabetes/T2DM, 251 were either refused to participate or excluded. We screened 119 (29.3\%) potential eligible controls with HbA1c test, and $29.4 \%$ (35/119) had HbA1c < 5.7\%, 34 were included in the final analyses. Among screened controls who were excluded $(\mathrm{n}=85)$, the median HbA1c was 6.0 [interquartile range (IQR) 5.8-6.2]. The majority of our study participants were African American (92.9\% among cases, 79.4\% among controls; Table 1). Cases were older (median age $=54, \mathrm{IQR} 49-60)$ than controls (median age $=51$, IQR $35-57)(\mathrm{p}=0.02)$. Daily smokers were more common among the controls $(35.3 \%)$ vs. cases $(24.7 \%)(\mathrm{p}=$ 0.02 ). The proportions of individuals with previous diagnosis of high cholesterol (70.6\% vs. 5.8\%) and high blood pressure $(70.8 \%$ vs. $35.3 \%)$, or obesity ( $66.3 \%$ vs. $35.3 \%)$ were greater among cases compared to controls $(\mathrm{p}<$ 0.05).

LTBI prevalence was higher among controls without T2DM $(14.7 \%, 5 / 34)$, compared to patients with newly diagnosed T2DM (9.2\%, 9/98; Table 2). After adjusting for age and gender, the odds of LTBI among cases was 0.45 times the odds among controls (95\% CI 0.13-1.71). The median HbA1c was $7.0 \%$ among cases with LTBI vs. $7.3 \%$ among cases without LTBI $(\mathrm{p}=0.75)$. The median HbA1c was $5.3 \%$ among controls with LTBI vs. $5.4 \%$ among controls without LTBI $(\mathrm{p}=0.37)$. Although nonsignificant, cases receiving metformin were less likely to have LTBI when compared to cases who were not receiving metformin [odds ratio (OR) $0.44,95 \% \mathrm{CI} 0.11-1.92$ ]. 
Table 1 Demographic and clinical characteristics of BATT study participants, Atlanta, Georgia 2016-2019 (N = 132)

\begin{tabular}{|c|c|c|c|c|}
\hline Characteristics & Controls N = 34 & Cases $\mathbf{N}=98$ & Total $\mathrm{N}=132$ & $\mathrm{X}^{2} \mathrm{p}$ value \\
\hline \multicolumn{5}{|l|}{ LTBI status } \\
\hline Negative & $29(85.3)$ & $89(90.8)$ & $118(89.4)$ & \multirow[t]{2}{*}{$0.35^{*}$} \\
\hline Positive & $5(14.7)$ & $9(9.2)$ & $14(10.6)$ & \\
\hline \multicolumn{5}{|l|}{ Age group } \\
\hline $21-40$ & $13(38.2)$ & $7(7.1)$ & $20(15.2)$ & \multirow[t]{3}{*}{$<0.01$} \\
\hline $41-60$ & $15(44.1)$ & $70(71.4)$ & $85(64.4)$ & \\
\hline$>60$ & $6(17.7)$ & $21(21.4)$ & $27(20.5)$ & \\
\hline \multicolumn{5}{|l|}{ Gender } \\
\hline Male & $17(50.0)$ & $42(42.9)$ & $59(44.7)$ & \multirow[t]{2}{*}{0.47} \\
\hline Female & $17(50.0)$ & $56(57.1)$ & $73(55.3)$ & \\
\hline \multicolumn{5}{|l|}{ Race/ethnicity } \\
\hline Non-hispanic white & $3(8.8)$ & $3(3.1)$ & $6(4.6)$ & \multirow[t]{5}{*}{$0.15^{*}$} \\
\hline Non-hispanic black & $27(79.4)$ & $91(92.9)$ & $118(89.4)$ & \\
\hline Hispanic & $1(2.9)$ & $1(1.0)$ & $2(1.5)$ & \\
\hline Asian or pacific islander & $1(2.9)$ & $1(1.0)$ & $2(1.5)$ & \\
\hline Other & $2(5.9)$ & $2(2.0)$ & $4(3.0)$ & \\
\hline \multicolumn{5}{|l|}{ Highest education } \\
\hline Less than high school & $8(23.5)$ & $18(18.4)$ & $26(19.7)$ & \multirow[t]{4}{*}{$0.15^{*}$} \\
\hline High school graduate & $15(44.1)$ & $60(61.2)$ & $75(56.8)$ & \\
\hline College/university & $8(23.5)$ & $18(18.4)$ & $26(19.7)$ & \\
\hline Graduate school & $3(8.8)$ & $2(2.0)$ & $5(3.8)$ & \\
\hline \multicolumn{5}{|c|}{ Ever lived with TB-sick person } \\
\hline No & $33(97.1)$ & $89(93.7)$ & $122(94.6)$ & \multirow[t]{3}{*}{$0.67^{*}$} \\
\hline Yes & $1(2.9)$ & $6(6.3)$ & $7(5.4)$ & \\
\hline Not sure & 0 & 3 & 3 & \\
\hline \multicolumn{5}{|c|}{ Ever told to have positive TST } \\
\hline No & $32(94.1)$ & $85(88.5)$ & $117(90.0)$ & \multirow[t]{3}{*}{$0.51^{*}$} \\
\hline Yes & $2(5.9)$ & $11(11.5)$ & $13(10.0)$ & \\
\hline Not sure & 0 & 2 & 2 & \\
\hline \multicolumn{5}{|l|}{ Current smoking } \\
\hline Daily & $12(35.3)$ & $24(24.7)$ & $36(27.5)$ & \multirow[t]{4}{*}{0.02} \\
\hline Less than daily & $6(17.7)$ & $5(5.2)$ & $11(8.4)$ & \\
\hline Not at all & $16(47.1)$ & $68(70.1)$ & $84(64.1)$ & \\
\hline Don't know/refused & 0 & 1 & 1 & \\
\hline \multicolumn{5}{|l|}{ Past smoking } \\
\hline Daily & $24(70.6)$ & $50(51.0)$ & $74(56.1)$ & \multirow[t]{3}{*}{0.12} \\
\hline Less than daily & $3(8.8)$ & $10(10.2)$ & $13(9.9)$ & \\
\hline Not at all & $7(20.6)$ & $38(38.8)$ & $46(34.1)$ & \\
\hline \multicolumn{5}{|l|}{ Alcohol consumption } \\
\hline Never & $18(52.9)$ & $65(66.3)$ & $83(62.9)$ & \multirow[t]{3}{*}{0.27} \\
\hline Moderate & $11(32.4)$ & $26(26.5)$ & $37(28.0)$ & \\
\hline Frequent & $5(14.7)$ & $7(7.1)$ & $12(9.1)$ & \\
\hline \multicolumn{5}{|c|}{ Ever diagnosed with high cholesterol level } \\
\hline No & $30(88.2)$ & $27(29.4)$ & $57(45.2)$ & \multirow[t]{3}{*}{$<0.01$} \\
\hline Yes & $4(5.8)$ & $65(70.6)$ & $69(54.8)$ & \\
\hline Not sure & 0 & 6 & 6 & \\
\hline
\end{tabular}

Among those with LTBI, increasing HbA1c level was $p=0.003$; Fig. 1). Among those with LTBI, nil count correlated with increasing QFT nil values $\left(R^{2}=0.547\right.$, increased on average by 0.064 (95\% CI $0.027-0.101)$ for 
Table 1 (continued)

\begin{tabular}{|c|c|c|c|c|}
\hline Characteristics & Controls $N=34$ & Cases $\mathbf{N}=98$ & Total $\mathbf{N}=132$ & $X^{2} p$ value \\
\hline \multicolumn{5}{|c|}{ Ever diagnosed with high blood pressure } \\
\hline No & $22(64.7)$ & $28(29.2)$ & $50(38.5)$ & \multirow[t]{3}{*}{$<0.01$} \\
\hline Yes & $12(35.3)$ & $68(70.8)$ & $80(61.5)$ & \\
\hline Not sure & 0 & 2 & 2 & \\
\hline \multicolumn{5}{|c|}{ Ever diagnosed with heart disease } \\
\hline No & $33(97.1)$ & $80(84.2)$ & $113(87.6)$ & \multirow[t]{3}{*}{$0.07^{*}$} \\
\hline Yes & $1(2.9)$ & $15(15.8)$ & $16(12.4)$ & \\
\hline Not sure & 0 & 3 & 3 & \\
\hline \multicolumn{5}{|c|}{ Ever diagnosed with liver disease } \\
\hline No & $32(94.1)$ & $90(96.8)$ & $122(96.1)$ & \multirow[t]{3}{*}{$0.61^{*}$} \\
\hline Yes & $2(5.9)$ & $3(3.2)$ & $5(3.9)$ & \\
\hline Not sure & 0 & 5 & 5 & \\
\hline \multicolumn{5}{|c|}{ Ever diagnosed with kidney disease } \\
\hline No & $34(100.0)$ & $88(92.6)$ & $122(94.6)$ & \multirow[t]{3}{*}{$0.19^{*}$} \\
\hline Yes & $0(0.0)$ & $7(7.3)$ & $7(5.4)$ & \\
\hline Not sure & 0 & 3 & 3 & \\
\hline \multicolumn{5}{|c|}{ Family members with T2DM } \\
\hline No & $7(20.6)$ & 29 (29.6) & $36(27.3)$ & \multirow[t]{2}{*}{0.31} \\
\hline Yes & $27(79.4)$ & $69(70.4)$ & $96(72.7)$ & \\
\hline \multicolumn{5}{|l|}{ BMI } \\
\hline Normal (18.5-25) & $12(35.3)$ & $11(11.2)$ & $23(17.4)$ & \multirow[t]{4}{*}{$<0.01 *$} \\
\hline Underweight $(<18.5)$ & $0(0.0)$ & $3(3.1)$ & $3(2.3)$ & \\
\hline Overweight (25-30) & $10(29.4)$ & $19(19.4)$ & $29(22.0)$ & \\
\hline Obese $(\geq 30)$ & $12(35.3)$ & $65(66.3)$ & $77(58.3)$ & \\
\hline \multicolumn{5}{|c|}{ Glycated hemoglobin level } \\
\hline Median (IQR) & $5.4(5.2-5.5)$ & $7.3(6.4-9.5)$ & $6.7(5.6-8.7)$ & $<0.01$ \\
\hline
\end{tabular}

Bold indicates that the finding is significant at $a=0.05$

$B M /$ body mass index; T2DM type-2 diabetes mellitus; IQR interquartile range; LTB/ latent tuberculosis infection; TB tuberculosis; TST tuberculin skin test * $\mathrm{p}$ value obtained from Fisher's exact tests

every unit increase in the HbA1c level (i.e., every one percentage point increase of $\mathrm{HbA1c}$ ). We did not observe a significant correlation between $\mathrm{HbA} 1 \mathrm{c}$ and TB antigennil $\left(R^{2}=0.139, p=0.190\right)$ or mitogen-nil $\left(R^{2}=0.002\right.$, $p=0.868$ ) values. For instance, for every unit increase in HbA1c level, the mitogen-nil value decreased on average by 0.005 (95\% CI $-0.070-0.060)$. Similarly, for every unit increase in HbA1c level, the TB antigen-nil decreased on average by 0.536 ( $95 \% \mathrm{CI}-1.377-0.304)$.

\section{Discussion}

We reported a high prevalence of LTBI among patients with newly diagnosed T2DM and healthy controls without T2DM in the metro Atlanta area. Our LTBI prevalence estimates $(9.2 \%$ among cases, $14.7 \%$ among controls) were higher compared to the US national (5.0\%) and US race-specific estimates among African Americans (5.3\%) [15]. Inconsistent with our findings, previous studies using nationally representative data from the US reported that diabetes is associated with increased odds of LTBI by one-three-fold [3, 4]. Another study from Atlanta conducted among recently arrived refugees also reported higher LTBI prevalence among participants with diabetes and pre-diabetes compared to euglycemic participants [1]. However, it is important to note that this hospital-based study, conducted primarily among African Americans, is consistent with recent US data that suggests the relationship between LTBI and T2DM differs across racial/ethnic groups [5].

Our findings also suggest that there may be regional differences in the relationship between LTBI and T2DM, which could be affected by background prevalences of both diseases or socioeconomic characteristics (i.e., an established risk factor for LTBI and T2DM) [2]. Of note, we reported more than $70 \%$ prevalence of undiagnosed prediabetes among screened family members/friends of cases. This finding is consistent with previously published studies reporting high prevalence of prediabetes/diabetes among 
Table 2 Crude and adjusted odds ratio of latent TB infection among BATT study participants, Atlanta, Georgia 2016-2019 ( $\mathrm{N}=132$ )

\begin{tabular}{|c|c|c|c|c|c|}
\hline \multirow[t]{2}{*}{ Characteristics } & \multicolumn{2}{|l|}{ LTBI status } & \multirow[t]{2}{*}{ Total $\mathrm{N}=132$} & \multirow[t]{2}{*}{ cOR $(95 \% \mathrm{Cl})$} & \multirow[t]{2}{*}{ aOR (95\% Cl) } \\
\hline & $\begin{array}{l}\text { Negative N (\%) = } \\
118(89.4)\end{array}$ & $\begin{array}{l}\text { Positive N (\%) = } 14 \\
\text { (10.6) }\end{array}$ & & & \\
\hline \multicolumn{6}{|l|}{ Group } \\
\hline Controls & $29(85.3)$ & $5(14.7)$ & $34(25.8)$ & Reference & Reference \\
\hline Cases (T2DM) & 89 (90.8) & $9(9.2)$ & $98(74.2)$ & $0.59(0.19-2.04)$ & $0.45(0.13-1.71)$ \\
\hline \multicolumn{6}{|l|}{ Age group } \\
\hline $21-40$ & $19(95.0)$ & $1(5.0)$ & $20(15.2)$ & $0.59(0.03-3.59)$ & $0.40(0.02-2.75)$ \\
\hline $41-60$ & $78(91.8)$ & $7(8.2)$ & $85(64.4)$ & Reference & Reference \\
\hline$>60$ & $21(77.8)$ & $6(22.2)$ & $27(20.5)$ & $3.18(0.94-10.61)$ & $3.09(0.89-10.44)$ \\
\hline \multicolumn{6}{|l|}{ Gender } \\
\hline Male & $52(88.1)$ & $7(11.9)$ & $59(44.7)$ & Reference & Reference \\
\hline Female & $66(90.4)$ & $5(9.6)$ & $73(55.3)$ & $0.79(0.26-2.44)$ & $1.09(0.34-3.48)$ \\
\hline \multicolumn{6}{|l|}{ Race/ethnicity } \\
\hline Non-hispanic black & $105(89.0)$ & $13(11.0)$ & $118(89.4)$ & $1.6(0.28-30.4)$ & \\
\hline Other & $13(92.9)$ & $1(7.1)$ & $14(10.6)$ & Reference & \\
\hline \multicolumn{6}{|l|}{ Highest education } \\
\hline Less than high school & $22(84.6)$ & $4(15.4)$ & $26(19.7)$ & Reference & \\
\hline High school graduate & $70(93.3)$ & $5(6.7)$ & $75(56.8)$ & $0.39(0.10-1.71)$ & \\
\hline College/university & $21(80.8)$ & $5(19.2)$ & $26(19.7)$ & $1.31(0.31-5.93)$ & \\
\hline Graduate school & $5(100.0)$ & $0(0.0)$ & $5(3.8)$ & N/A & \\
\hline \multicolumn{6}{|c|}{ Ever lived with TB-sick person } \\
\hline No & $110(90.2)$ & $12(9.8)$ & $122(94.6)$ & Reference & \\
\hline Yes & $5(71.4)$ & $2(28.6)$ & $7(5.4)$ & $3.67(0.49-19.20)$ & \\
\hline Not sure & 3 & 0 & 3 & & \\
\hline \multicolumn{6}{|c|}{ Currently on metformin, among cases $(\mathrm{N}=98)$} \\
\hline No & $23(85.2)$ & $4(14.8)$ & $27(27.8)$ & Reference & \\
\hline Yes & $65(92.9)$ & $5(7.1)$ & 70 (72.2) & $0.44(0.11-1.92)$ & \\
\hline Missing & 1 & 0 & 1 & & \\
\hline \multicolumn{6}{|l|}{ Current smoking } \\
\hline No & $76(90.5)$ & $8(9.5)$ & $84(64.1)$ & Reference & \\
\hline Yes & $41(87.2)$ & $6(12.8)$ & $47(35.9)$ & $1.39(0.43-4.27)$ & \\
\hline Missing & 1 & 1 & 2 & & \\
\hline \multicolumn{6}{|l|}{ Past smoking } \\
\hline No & $44(97.8)$ & $1(2.2)$ & $45(34.1)$ & Reference & \\
\hline Yes & $74(85.1)$ & $13(14.9)$ & $87(65.9)$ & $7.73(1.46-142.75)$ & \\
\hline \multicolumn{6}{|l|}{ Alcohol consumption } \\
\hline Never & $75(90.4)$ & $8(9.6)$ & $83(62.9)$ & Reference & \\
\hline Moderate & $32(86.5)$ & $5(13.5)$ & $37(28.0)$ & $1.47(0.42-4.74)$ & \\
\hline Frequent & $11(91.7)$ & $1(8.3)$ & $12(9.1)$ & $0.85(0.04-5.34)$ & \\
\hline \multicolumn{6}{|c|}{ Ever diagnosed with high cholesterol level } \\
\hline No & $50(87.7)$ & $7(12.3)$ & $57(45.2)$ & Reference & \\
\hline Yes & $62(89.9)$ & $7(10.1)$ & $69(54.8)$ & $0.81(0.26-2.50)$ & \\
\hline Not sure & 6 & 0 & 6 & & \\
\hline \multicolumn{6}{|c|}{ Ever diagnosed with high blood pressure } \\
\hline No & $47(94.0)$ & $3(6.0)$ & $50(38.5)$ & Reference & \\
\hline Yes & $69(86.3)$ & $11(13.8)$ & $80(61.5)$ & $2.50(0.73-11.48)$ & \\
\hline Not sure & 2 & 0 & 2 & & \\
\hline \multicolumn{6}{|c|}{ Ever diagnosed with heart disease } \\
\hline No & $101(89.4)$ & $12(10.6)$ & $113(87.6)$ & Reference & \\
\hline
\end{tabular}


Table 2 (continued)

\begin{tabular}{|c|c|c|c|c|c|}
\hline \multirow[t]{2}{*}{ Characteristics } & \multicolumn{2}{|l|}{ LTBI status } & \multirow[t]{2}{*}{ Total $\mathbf{N}=132$} & \multirow[t]{2}{*}{ cOR $(95 \% \mathrm{Cl})$} & \multirow[t]{2}{*}{ aOR $(95 \% \mathrm{Cl})$} \\
\hline & $\begin{array}{l}\text { Negative } N(\%)= \\
118(89.4)\end{array}$ & $\begin{array}{l}\text { Positive N }(\%)=14 \\
(10.6)\end{array}$ & & & \\
\hline Yes & $14(87.5)$ & $2(12.5)$ & $16(12.4)$ & $1.20(0.18-5.05)$ & \\
\hline Not sure & 3 & 0 & 3 & & \\
\hline \multicolumn{6}{|c|}{ Ever diagnosed with liver disease } \\
\hline No & $109(89.3)$ & $13(10.7)$ & $122(96.1)$ & Reference & \\
\hline Yes & $4(80.0)$ & $1(20.0)$ & $5(3.9)$ & $2.10(0.10-15.57)$ & \\
\hline Not sure & 5 & 0 & 5 & & \\
\hline \multicolumn{6}{|c|}{ Ever diagnosed with kidney disease } \\
\hline No & $108(88.5)$ & $14(11.5)$ & $122(94.6)$ & Reference & \\
\hline Yes & $7(100.0)$ & $0(0.0)$ & $7(5.4)$ & N/A & \\
\hline Not sure & 3 & 0 & 3 & & \\
\hline \multicolumn{6}{|c|}{ Family members with T2DM } \\
\hline No & $29(80.6)$ & $7(19.4)$ & $36(27.3)$ & Reference & \\
\hline Yes & $89(92.7)$ & $7(7.3)$ & $96(72.7)$ & $0.33(0.10-1.03)$ & \\
\hline \multicolumn{6}{|l|}{ BMI } \\
\hline Normal (18.5-25) & $19(82.6)$ & $4(17.4)$ & $23(17.4)$ & Reference & \\
\hline Underweight (< 18.5) & $3(100.0)$ & $0(0.0)$ & $3(2.3)$ & $\mathrm{N} / \mathrm{A}$ & \\
\hline Overweight (25-30) & 28 (96.6) & $1(3.5)$ & $29(22.0)$ & $0.17(0.01-1.26)$ & \\
\hline Obese $(\geq 30)$ & $68(88.3)$ & $9(11.7)$ & $77(58.3)$ & $0.63(0.18-2.52)$ & \\
\hline \multicolumn{6}{|c|}{ Glycated hemoglobin level } \\
\hline Median (IQR) & $6.7(5.6-8.6)$ & $6.0(5.3-9.2)$ & $6.7(5.6-8.7)$ & & \\
\hline
\end{tabular}

aOR adjusted odds ratio; $B M I$ body mass index; $C I$ confidence interval; $C O R$ crude odds ratio; T2DM type-2 diabetes mellitus; IQR interquartile range

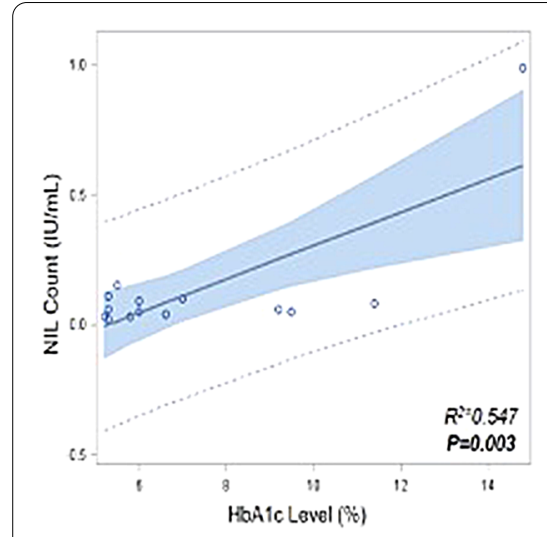

a

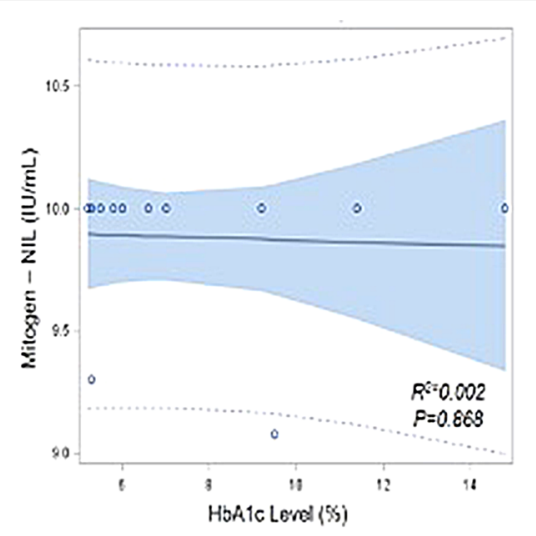

b

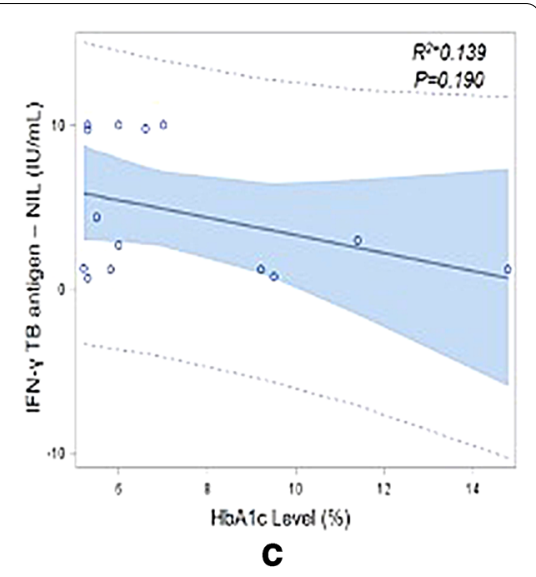

C

\begin{tabular}{|c|c|c|}
\hline Outcomes & $\beta$-estimates for HbA1c & $95 \% \mathrm{Cl}$ for $\beta$ \\
\hline Nil Count & 0.064 & $0.027,0.101$ \\
\hline Mitogen - Nil & -0.005 & $-0.070,0.060$ \\
\hline TB Antigen - Nil & -0.536 & $-1.377,0.304$ \\
\hline
\end{tabular}

Fig. 1 Results from linear regression of nil count, mitogen-nil, and TB antigen-nil by HbA1c level among patients with positive QFT, $N=14$ 
household/family members of individuals with T2DM [16-18]. Our findings also support previous recommendations suggesting that friends or family members of patients with T2DM would benefit from T2DM screening in an effort to introduce early intervention to prevent T2DM development.

Among individuals with LTBI, we reported a positive correlation between HbA1c and the QFT negative control without antigens or mitogens (nil count). The nil value is used to determine if patient has a pre-existing nonspecific immune response which could lead to a falsepositive. A cross-sectional study among US adults with LTBI reported a higher IFN- $\gamma$ antigen response among those with pre-diabetes compared to euglycemic adults, but found that the average nil value was similar among patients with diabetes, prediabetes, and euglycemia [19]. To date, the relationship between the quantitative IFN- $\gamma$ responses specific to $M t b$ and T2DM is inconclusive. We did not observe a significant correlation between glycemic control with TB antigen or mitogen responses, although we only analyzed this relationship in 14 study participants. Similar to our findings, a cross-sectional study conducted in Indonesia from 2014 to 2015 reported no significant difference of median TB antigen-nil value across different HbA1c levels (HbA1c < 7.0\% vs. 7.0-9.9\% vs. $\geq 10.0 \%$; $=0.73$ ) [20]. However, a 2014 cross-sectional study conducted in India reported that individuals with LTBI and T2DM had decreased mean of $M t b$ antigen-stimulated (net cytokines) levels including lower IFN- $\gamma(10.5 \mathrm{pg} / \mathrm{mL}$ vs. $249.2 \mathrm{pg} / \mathrm{mL})$, TNF- $\alpha(6.5 \mathrm{pg} / \mathrm{mL}$ vs. $328.1 \mathrm{pg} / \mathrm{mL})$, interleukin (IL)-17A (14.2 $\mathrm{pg} / \mathrm{mL}$ vs. $24.4 \mathrm{pg} / \mathrm{mL})$, and IL-10 (95.6 pg/mL vs. $220.6 \mathrm{pg} / \mathrm{mL})$ when compared to LTBI individuals without T2DM [21]. Further studies to better characterize the relationship between T2DM, hyperglycemia, and immune responses specific to LTBI are still warranted.

\section{Conclusion}

In conclusion, we reported a high prevalence of LTBI among adults with T2DM and family members without T2DM. Although we did not observe a significant association between LTBI prevalence and T2DM we did observe a positive correlation between $\mathrm{HbA} 1 \mathrm{c}$ and nil count among individuals with LTBI. Larger prospective investigations across different regions and race/ethcity subgroups are warranted to determine the role of LTBI in pre-diabetes/T2DM risk. Further studies that measure LTBI prevalence among household members living with patients with T2DM are also needed to determine whether household/family members of individuals with T2DM could be considered as a priority target group for LTBI screening.

\section{Limitations}

Our study was subject to several limitations. First, we had a small sample size enrolled from a single hospital and new diabetes cases were diagnosed within the past three years. Thus, our results may not be widely generalizable to other settings or other new diabetes patients. However, we used 3 years cut-off to define newly diagnosed T2DM due to the long natural progression of T2DM [22, 23] and the potential delay in receiving T2DM diagnosis among our study population, of which the majority came from lower socioeconomic levels [24, 25]. Second, our study was designed to assess the association between LTBI and the risk of T2DM. The non-significant findings we reported in this manuscript may in part be due to a bi-directional relationship between LTBI and T2DM where T2DM increases the risk of LTBI. Third, because we were unable to compare at what age cases or controls were exposed to MTB, the higher prevalence of LTBI among controls may be a reflection of when during their lifecourse they were initially infected with LTBI. Last, we enrolled a smaller number of controls compared to cases, which may due to our control selection strategy (i.e., friends or family members vs. community-based recruitment). It is plausible that friends/ family members shared lifestyle or other modifiable T2DM risk factors including diet, physical activity, or smoking $[18,26]$, which could lead to pre-diabetes (HbA1c $\geq$ $5.7 \%$ ) or previous T2DM diagnosis and exclusion from this study. Previous studies highlighted that selecting friends/ family as controls may pose several epidemiologic challenges including potential case-control overmatch, similarity in responding study's questionnaires, and potential bias among cases in nominating their controls [27]. However, we believe that using friends or family members as controls is still a viable and cost-efficient option for a small pilot project like the present study.

\begin{abstract}
Abbreviations
aOR: Adjusted odds ratio; Cl: Confidence interval; cOR: Crude odds ratio; HbA1c: Glycated hemoglobin; HIV: Human immunodeficiency virus; IFN- $\gamma$ : Interferon gamma; IL: Interleukin; IQR: Interquartile range; LTBI: Latent tuberculosis infection; Mtb: Mycobacterium tuberculosis; OR: Odds ratio; QFT: Quantiferon; T2DM: Type-2 diabetes mellitus; TB: Tuberculosis; TNF-a: Tumor necrosis factor alpha; US: United States of America.
\end{abstract}

\section{Supplementary Information}

The online version contains supplementary material available at https://doi. org/10.1186/s13104-021-05662-0.

Additional file 1: Figure S1. Study enrollment diagram.

\section{Acknowledgements}

We would like to thank Marissa Baron, MPH; Susanna Trost, MPH; and Toby Akingbade, MPH for their assistance during the study enrollment period. 


\section{Authors' contributions}

MJM, JSH, GEU, and ADS conceived the study design. ADS, GAA, and JTA obtained the data. ADS and MJM performed the analyses and wrote the first draft of the manuscript. ADS, JSH, GAA, JTA, GEU, and MJM reviewed, and revised the final version of the manuscript. All authors read and approved the final manuscript.

\section{Funding}

This work was supported by the National Center for Advancing Translation Sciences of the National Institutes of Health $(\mathrm{NIH})$ under award numbers UL1TR000454 and NIH R03AI135388. The content is solely the responsibility of the authors and does not necessarily represent the official views of the National Institutes of Health.

\section{Availabillity of data and materials}

The datasets generated and/or analysed during the current study are not publicly available due to the confidential nature of patients' data but are available from the corresponding author on reasonable request.

\section{Declarations}

\section{Ethics approval and consent to participate}

This study was submitted to, reviewed, and approved by the Institutional Review Boards (IRBs) at Emory University (IRB \#85070), Georgia State Universities (IRB \#H16371) as well as Grady Research Oversight Committee, Atlanta, USA. Written informed consent was obtained from eligible participants after conducting a consent discussion led by BATT study staff.

\section{Consent for publication}

N/A.

\section{Competing interests}

We have no competing interests to declare.

\section{Author details}

${ }^{1}$ Division of Epidemiology and Biostatistics, Department of Population Health Sciences, School of Public Health, Georgia State University, Atlanta, GA 30303, USA. ${ }^{2}$ Division of Endocrinology, Metabolism and Lipids, Emory University School of Medicine, Atlanta, GA, USA. ${ }^{3}$ Hubert Department of Global Health, Rollins School of Public Health, Emory University, Atlanta, GA, USA.

Received: 22 February 2021 Accepted: 17 June 2021

Published online: 30 June 2021

\section{References}

1. Hensel RL, Kempker RR, Tapia J, Oladele A, Blumberg HM, Magee MJ. Increased risk of latent tuberculous infection among persons with prediabetes and diabetes mellitus. Int J Tuberc Lung Dis. 2016;20(1):71-8.

2. Lee MR, Huang YP, Kuo YT, Luo CH, Shih YJ, Shu CC, et al. Diabetes mellitus and latent tuberculosis infection: a systemic review and meta analysis. Clin Infect Dis. 2017:64(6):719-27.

3. Barron MM, Shaw KM, Bullard KM, Ali MK, Magee MJ. Diabetes is associated with increased prevalence of latent tuberculosis infection: findings from the National Health and Nutrition Examination Survey, 2011-2012. Diabetes Res Clin Pract. 2018:139:366-79.

4. Martinez L, Zhu L, Castellanos ME, Liu Q, Chen C, Hallowell BD, et al. Glycemic control and the prevalence of tuberculosis infection: a populationbased observational study. Clin Infect Dis. 2017;65(12):2060-8.

5. Haddad MB, Lash TL, Castro KG, Hill AN, Navin TR, Gandhi NR, et al. Tuberculosis infection among people with diabetes: United States population differences by race/ethnicity. Am J Prev Med. 2020;58(6):858-63.

6. Magee MJ, Salindri AD, Gujral UP, Auld SC, Bao J, Haw JS, et al. Convergence of non-communicable diseases and tuberculosis: a two-way street? Int J Tuberc Lung Dis. 2018;22(11):1258-68.

7. Pal R, Ansari MA, Hameed S, Fatima Z. Diabetes mellitus as hub for tuberculosis infection: a snapshot. Int J Chronic Dis. 2016:2016:5981574.
8. Martinez N, Kornfeld $\mathrm{H}$. Tuberculosis and diabetes: from bench to bedside and back. Int J Tuberc Lung Dis. 2019;23(6):669-77.

9. Martinez N, Cheng CY, Ketheesan N, Cullen A, Tang Y, Lum J, et al. mTORC2/Akt activation in adipocytes is required for adipose tissue inflammation in tuberculosis. EBioMedicine. 2019:45:314-27.

10. Podell BK, Ackart DF, Obregon-Henao A, Eck SP, Henao-Tamayo M, Richardson M, et al. Increased severity of tuberculosis in Guinea pigs with type 2 diabetes: a model of diabetes-tuberculosis comorbidity. Am J Pathol. 2014;184(4):1104-18.

11. van Raalten $F$, Hiemstra $Y L$, Keulen $N$, van Duivenvoorde $Y$, Stoecklein $K$, Verhagen EA, et al. Level of agreement of point-of-care and laboratory $\mathrm{HbA} 1 \mathrm{c}$ measurements in the preoperative outpatient clinic in nondiabetic patients who are overweight or obese. J Clin Monit Comput. 2019;33(6):1139-44.

12. American Diabetes Association. Diagnosis and classification of diabetes mellitus. Diabetes Care. 2014;37(Suppl 1):S81-90.

13. Mazurek GH, Villarino ME. Guidelines for using the QuantiFERON_TB test for diagnosing latent Mycobacterium tuberculosis infection. MMWR Recomm Rep. 2003;52(RR02):15-8.

14. Greenland S, Pearl J, Robins JM. Causal diagrams for epidemiologic research. Epidemiology. 1999;10(1):37-48.

15. Miramontes R, Hill AN, Woodruff YRS, Lambert LA, Navin TR, Castro KG et al. Tuberculosis infection in the United States: prevalence estimates from the national health and nutrition examination survey, 2011-2012. PLOS ONE. 2015;10(11):e0140881.

16. Ramaswamy G, Chinnakali P, Selvaraju S, Nair D, Thekkur P, Selvaraj K, et al. High prevalence of prediabetes among the family members of individuals with diabetes. Findings from targeted screening program from south India. Diabetes Metab Syndr. 2019;13(1):866-72.

17. Jali MV, Kambar S. Prevalence of diabetes amongst the family members of known diabetics. Int J Diab Dev Ctries. 2006;26(2):81-5.

18. InterAct C, Scott RA, Langenberg C, Sharp SJ, Franks PW, Rolandsson O, et al. The link between family history and risk of type 2 diabetes is not explained by anthropometric, lifestyle or genetic risk factors: the EPICInterAct study. Diabetologia. 2013:56(1):60-9.

19. Magee MJ, Trost SL, Salindri AD, Amere G, Day CL, Gandhi NR. Adults with Mycobacterium tuberculosis infection and pre-diabetes have increased levels of QuantiFERON interferon-gamma responses. Tuberculosis. 2020;122:101935

20. Koesoemadinata RC, MCAllister SM, Soetedjo NNM, Febni Ratnaningsih D, Ruslami R, Kerry S, et al. Latent TB infection and pulmonary TB disease among patients with diabetes mellitus in Bandung, Indonesia. Trans R Soc Trop Med Hyg. 2017;111(2):81-9.

21. Kumar NP, George PJ, Kumaran P, Dolla CK, Nutman TB, Babu S. Diminished systemic and antigen-specific type 1, type 17, and other proinflammatory cytokines in diabetic and prediabetic individuals with latent Mycobacterium tuberculosis infection. J Infect Dis. 2014;210(10):1670-8.

22. Sagesaka H, Sato Y, Someya Y, Tamura Y, Shimodaira M, Miyakoshi T, et al. Type 2 diabetes: when does it start? J Endocr Soc. 2018;2(5):476-84.

23. Ferrannini E, Nannipieri M, Williams K, Gonzales C, Haffner SM, Stern MP. Mode of onset of type 2 diabetes from normal or impaired glucose tolerance. Diabetes. 2004;53(1):160-5.

24. Samuels TA, Cohen D, Brancati FL, Coresh J, Kao WH. Delayed diagnosis of incident type 2 diabetes mellitus in the ARIC study. Am J Manag Care. 2006;12(12):717-24

25. Roche MM, Wang PP. Factors associated with a diabetes diagnosis and late diabetes diagnosis for males and females. J Clin Transl Endocrinol. 2014;1(3):77-84.

26. Baig AA, Benitez A, Quinn MT, Burnet DL. Family interventions to improve diabetes outcomes for adults. Ann NY Acad Sci. 2015;1353(1):89-112.

27. Zhong C, Cockburn M, Cozen W, Voutsinas J, Lacey JV, Luo J, et al. Evaluating the use of friend or family controls in epidemiologic case-control studies. Cancer Epidemiol. 2017;46:9-13.

\section{Publisher's Note}

Springer Nature remains neutral with regard to jurisdictional claims in published maps and institutional affiliations. 